Dom. Cien., ISSN: 2477-8818

Vol. 3, núm. 1, enero, 2017, pp. 432-442

\title{
Síndrome de Burnout en estudiantes de Odontología
}

\author{
Burnout Syndrome in Dental Students
}

Síndrome de Burnout em Estudantes de Odontologia

\author{
R. Peralta-Ayala \\ cari_cperalta@hotmail.com \\ Tamara J. Moya Silva" \\ tjmoya@uce.edu.ec
}

Recibido: 20 de noviembre de 2016 * Corregido: 16 de diciembre de 2016 * Aceptado: 7 de enero de 2017

'Odontólogo, Facultad de Odontología, Universidad Central del Ecuador, Quito, Ecuador.

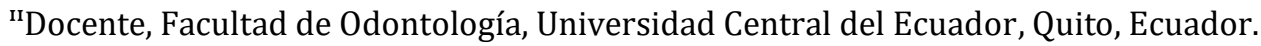




\section{Resumen.}

Objetivo: Evaluar el grado de afección de Síndrome de Burnout en los estudiantes de octavo y noveno semestre de la Facultad de Odontología de la Universidad Central del Ecuador. Materiales y métodos: Se realizó un estudio de tipo transversal, descriptivo, analítico y cuantitativo, según la edad, género, estado civil cargas familiares y si presentan trabajo extracurricular, mediante el test de Maslach y Jackson (1981), quienes realizaron el MBI - Human Services Survey, dirigido a los profesionales de la salud. Este instrumento es la versión clásica del MBI. Está constituido por 22 ítems que se distribuyen en 3 escalas para evaluar la frecuencia con que los profesionales perciben: Baja realización personal 8 ítems, agotamiento emocional 9 ítems, despersonalización 5 ítems, los resultados fueron procesados y tabulados por el paquete estadístico SPPS versión 20 se aplicó el test estadístico de la prueba del Chi cuadrado de Pearson. Resultados: No se encontraron diferencias significativas con la variable de estudio género, se demostró un nivel alto en la variable edades con intervalo de 20 a 25 años $(15,7 \%)$, se encontraron niveles altos de cansancio emocional por el estado civil en la unión libre 50\%, se tiene una mayor proporción que si tienen cargas familiares $(21,9 \%)$ y nivel bajo se tiene en la variable que no tienen trabajo $(18,8 \%)$. Conclusiones: Existen muy pocos estudios del Burnout aplicado a los estudiantes de la Facultad de Odontología de la Universidad Central del Ecuador y no se define diferencias el síndrome en este tipo de estudiantes con respecto a los grupos estudiados.

Palabras clave: Síndrome de burnout; estudiantes; universidad; salud mental. 


\section{Abstract.}

Objective: To evaluate the degree of affection of Burnout Syndrome in the eighth and ninth semester students of the Faculty of Dentistry of the Central University of Ecuador. Materials and Methods: A cross - sectional, descriptive, analytical and quantitative study was carried out, according to age, gender, family status, and if they present extracurricular work, using the Maslach and Jackson test (1981) Human Services Survey, aimed at health professionals. This instrument is the classic MBI version. It consists of 22 items that are distributed in 3 scales to evaluate the frequency with which professionals perceive: Low personal accomplishment 8 items, emotional exhaustion 9 items, 5 items depersonalization, the results were processed and tabulated by the SPPS version 20 statistical package Applied the statistical test of the Pearson Chi square test. Results: No significant differences were found with the gender study variable, a high level was found in the variable ages ranging from 20 to 25 years (15.7\%). There were high levels of emotional exhaustion due to marital status in the Free union $50 \%$, has a greater proportion than if they have family burdens $(21.9 \%)$ and low level has in the variable that do not have work (18.8\%). Conclusions: There are very few studies of Burnout applied to students of the Faculty of Dentistry of the Central University of Ecuador and there are no differences in the syndrome in this type of students with respect to the studied groups

Key Words: Burnout síndrome; students; university; mental health. 


\section{Resumo.}

Objetivo: Avaliar o grau de afeição da Síndrome de Burnout no semestre os alunos oitavo e nono da Faculdade de Odontologia da Universidade Central do Equador. Materiais e Métodos: A cruz seccionais, descritivos, analíticos e estudo quantitativo foi realizado, de acordo com idade, sexo, situação familiar, e se apresentar o trabalho extracurricular, utilizando o teste de Maslach e Jackson (1981) Human Services Survey Visando profissionais de saúde. Este instrumento é a versão clássica MBI. É composto de 22 itens que são distribuídos em três escalas para avaliar a frequência com que profissionais percebem: Low realização pessoal 8 itens, exaustão emocional 9 itens, 5 itens despersonalização, os resultados foram processados e tabulados pela versão SPPS 20 pacote estatístico aplicadas as teste estatístico do teste quadrado de Pearson Chi. Resultados: Nenhuma diferença significativa estavam com o estudo de gênero encontrada variável, um nível elevado foi encontrado nas idades variando de 20 a 25 anos (15,7\%). Eram altos níveis de exaustão emocional devido ao estado civil na União gratuito $50 \%$, você tem uma proporção maior do que se eles têm encargos familiares $(21,9 \%)$ e de baixo nível tem na variável que não tem trabalho $(18,8 \%)$. Conclusões: Há muito poucos estudos sobre Burnout aplicada a estudantes da Faculdade de Odontologia da Universidade Central do Equador e não existem diferenças na síndrome neste tipo de estudantes com relação aos grupos estudados

Palavras chave: Síndrome de burnout; Estudantes; universidade; saúde mental.

\footnotetext{
435 Vol. 3, núm. 1, enero, 2017, pp. 432-442
Carina R. Peralta-Ayala; Tamara J. Moya Silva
} 


\section{Introducción.}

La sociedad en la actualidad se caracteriza por presentar un alto nivel de exigencias y competitividad, además los cambios en las organizaciones y la actividad laboral tienden a producir una mayor mentalización del trabajo, por lo que aumenta la incertidumbre en la solución de problemas propios del trabajo. (1)

La necesidad de estudio del Síndrome de Burnout, es actualmente una patología derivada del trabajo y es precursor del deterioro de la salud mental.

El síndrome de Burnout afecta principalmente a profesionales de la salud, que brindan un servicio de ayuda y que requieren contacto directo con las personas, y es considerado un trastorno de adaptación al medio, debido a las exigencias sociales, psicológicas, económicas, académicas, laborales por un tiempo prolongado, que afecta el estilo de vida de la persona, y disminuye la calidad asistencial.

El síndrome de Burnout en odontólogos se considera como un riego profesional importante, ya que son los más propensos a desarrollarlo, debido a la naturaleza de su trabajo, es por esto que la Odontología es calificada como una de las profesiones más estresantes en el campo de la salud.

Mediante esta investigación se pretende ayudar y colaborar con los profesionales en desarrollo como son los estudiantes de octavo y noveno semestre de la Facultad de Odontología de la Universidad Central, para que estén informados sobre el síndrome de Burnout, sus causas, consecuencias y cómo pueden evitarlo. 


\section{Materiales y métodos.}

El estudio que se va a realizar es transversal, descriptivo, analítico y cuantitativo, ya que los resultados obtenidos serán examinados de forma numérica en el que pueda obtenerse un conteo específico de los datos.

La presente investigación se realizó Facultad de Odontología de la Universidad Central del Ecuador, en los alumnos que estuvieron cursando octavo y noveno semestre, en el área de clínicas de pregrado.

La población que se investigó es finita que tiene un objeto de estudio bien definido.

Los estudiantes encuestados fueron 211, de los cuales corresponden 123 estudiantes de octavo semestre y 88 estudiantes de noveno semestre.

Se incluirá en el estudio a todos los estudiantes de octavo y noveno semestre que realicen prácticas en la clínica integral y de odontopediatría, durante el periodo de abril a agosto de 2014, que deseen ser partícipes de la investigación.

Se excluirá a los estudiantes que no deseen ser integrantes del proyecto de investigación, y aquellos estudiantes que ya hayan sido detectados o diagnosticados con Síndrome de Burnout. 
Dom. Cien., ISSN: 2477-8818

Vol. 3, núm. 1, enero, 2017, pp. 432-442

\section{Resultados.}

\begin{tabular}{cccccccccc} 
& \multicolumn{10}{c}{ NIVELES } \\
\cline { 2 - 10 } CANSANCIO EMOCIONAL & \multicolumn{1}{c}{ DESPERSONALIZACIÓN } & \multicolumn{3}{c}{ REALIZACIÓN } \\
GENERO & BAJO & MEDIO & ALTO & BAJO & MEDIO & ALTO & BAJO & MEDIO & ALTO \\
MASCULINO & $11,1 \%$ & $77,8 \%$ & $11,1 \%$ & $57,8 \%$ & $40,0 \%$ & $2,2 \%$ & - & $73,3 \%$ & $26,7 \%$ \\
FEMENINO & $15,7 \%$ & $76,5 \%$ & $7,8 \%$ & $68,1 \%$ & $31,3 \%$ & $0,6 \%$ & - & $63,9 \%$ & $36,1 \%$ \\
TOTAL & $14,7 \%$ & $76,8 \%$ & $8,5 \%$ & $65,9 \%$ & $33,2 \%$ & $0,9 \%$ & - & $65,9 \%$ & $34,1 \%$ \\
\hline PRUEBA CHI & \multicolumn{1}{c}{ INDEPENDENCIA } & \multicolumn{3}{c}{ INDEPENDENCIA } & & INDEPENDENCIA \\
CUADRADO & \multicolumn{2}{c}{$\mathrm{p}=0,621$} & \multicolumn{3}{c}{$\mathrm{p}=0,308$} & & $\mathrm{p}=0,234$
\end{tabular}

Tabla $N^{\circ}$ 1.- Análisis de datos de la encuesta por género

La prueba Chi cuadrado determina que las proporciones no son significativamente diferentes como para generar dependencia de alguno de los géneros sobre los niveles.

NIVELES

\begin{tabular}{|c|c|c|c|c|c|c|c|c|c|}
\hline \multirow[b]{3}{*}{ EDADES } & \\
\hline & \multicolumn{3}{|c|}{ CANSANCIO EMOCIONAL } & \multicolumn{3}{|c|}{ DESPERSONALIZACIÓN } & \multicolumn{3}{|c|}{ REALIZACIÓN } \\
\hline & ВАJO & MEDIO & ALTO & ВАJO & MEDIO & ALTO & ВАJO & MEDIO & ALTO \\
\hline 20 a 24 & $14,9 \%$ & $80,1 \%$ & $5,0 \%$ & $67,4 \%$ & $31,2 \%$ & $1,4 \%$ & - & $67,4 \%$ & $32,6 \%$ \\
\hline 25 a 30 & $14,3 \%$ & $70,0 \%$ & $15,7 \%$ & $62,9 \%$ & $37,1 \%$ & $0,0 \%$ & - & $62,9 \%$ & $37,1 \%$ \\
\hline TOTAL & $14,7 \%$ & $76,8 \%$ & $8,5 \%$ & $65,9 \%$ & $33,2 \%$ & $0,9 \%$ & - & $65,9 \%$ & $34,1 \%$ \\
\hline PRUEBA CHI & \multicolumn{3}{|c|}{ DEPENDENCIA } & \multicolumn{3}{|c|}{ INDEPENDENCIA } & \multicolumn{3}{|c|}{ INDEPENDENCIA } \\
\hline CUADRADO & \multicolumn{3}{|c|}{$\mathrm{p}=0,031$} & \multicolumn{3}{|c|}{$\mathrm{p}=0,442$} & \multicolumn{3}{|c|}{$\mathrm{p}=0,515$} \\
\hline
\end{tabular}

Tabla $N^{\circ}$ 2.- Análisis de datos de la encuesta por edades

En el cansancio emocional se observa que la prueba Chi cuadrado indica dependencia, esto se debe a que en el nivel alto se tiene una mayor proporción de encuestados en el intervalo de 20 a 25 años $(15,7 \%)$. 


\section{NIVELES}

\begin{tabular}{|c|c|c|c|c|c|c|c|c|c|}
\hline \multirow[b]{2}{*}{ ESTADO CIVIL } & \multicolumn{3}{|c|}{ CANSANCIO EMOCIONAL } & \multicolumn{3}{|c|}{ DESPERSONALIZACIÓN } & \multicolumn{3}{|c|}{ REALIZACIÓN } \\
\hline & BAJO & MEDIO & ALTO & ВАJO & MEDIO & ALTO & BAJO & MEDIO & ALTO \\
\hline Soltero & $16,6 \%$ & $77,7 \%$ & $5,7 \%$ & $66,3 \%$ & $32,6 \%$ & $1,1 \%$ & - & $65,1 \%$ & $34,9 \%$ \\
\hline Casado & $6,5 \%$ & $74,2 \%$ & $19,4 \%$ & $61,3 \%$ & $38,7 \%$ & $0,0 \%$ & - & $67,7 \%$ & $32,3 \%$ \\
\hline Divorciado & $0,0 \%$ & $100,0 \%$ & $0,0 \%$ & $100,0 \%$ & $0,0 \%$ & $0,0 \%$ & - & $100,0 \%$ & $0,0 \%$ \\
\hline Unión libre & $0,0 \%$ & $50,0 \%$ & $50,0 \%$ & $75,0 \%$ & $25,0 \%$ & $0,0 \%$ & - & $75,0 \%$ & $25,0 \%$ \\
\hline TOTAL & $14,7 \%$ & $76,8 \%$ & $8,5 \%$ & $65,9 \%$ & $33,2 \%$ & $0,9 \%$ & - & $65,9 \%$ & $34,1 \%$ \\
\hline PRUEBA CHI & \multicolumn{3}{|c|}{ DEPENDENCIA } & \multicolumn{3}{|c|}{ INDEPENDENCIA } & \multicolumn{3}{|c|}{ INDEPENDENCIA } \\
\hline CUADRADO & \multicolumn{3}{|c|}{$\mathrm{p}=0,009$} & \multicolumn{3}{|c|}{$\mathrm{p}=0,962$} & \multicolumn{3}{|c|}{$\mathrm{p}=0,860$} \\
\hline
\end{tabular}

Tabla $N^{\circ}$ 3.- Análisis de datos de la encuesta por estado civil

\begin{tabular}{|c|c|c|c|c|c|c|c|c|c|}
\hline \multirow[b]{3}{*}{$\begin{array}{c}\text { CARGAS } \\
\text { FAMILIARES }\end{array}$} & \multicolumn{9}{|c|}{$\begin{array}{c}\text { NIVELES } \\
\end{array}$} \\
\hline & \multicolumn{3}{|c|}{ CANSANCIOEMOCIONAL } & \multicolumn{3}{|c|}{ DESPERSONALIZACIÓN } & \multicolumn{3}{|c|}{ REALIZACIÓN } \\
\hline & BAJO & MEDIO & ALTO & BAJO & MEDIO & ALTO & BAJO & MEDIO & ALTO \\
\hline SI & $9,4 \%$ & $68,8 \%$ & $21,9 \%$ & $71,9 \%$ & $28,1 \%$ & $0,0 \%$ & - & $59,4 \%$ & $40,6 \%$ \\
\hline NO & $15,6 \%$ & $78,2 \%$ & $6,1 \%$ & $64,8 \%$ & $34,1 \%$ & $1,1 \%$ & - & $67,0 \%$ & $33,0 \%$ \\
\hline TOTAL & $14,7 \%$ & $76,8 \%$ & $8,5 \%$ & $65,9 \%$ & $33,2 \%$ & $0,9 \%$ & - & $65,9 \%$ & $34,1 \%$ \\
\hline PRUEB A CHI & \multicolumn{3}{|c|}{ DEPENDENCIA } & \multicolumn{3}{|c|}{ INDEPENDENCIA } & \multicolumn{3}{|c|}{ INDEPENDENCIA } \\
\hline CUADRADO & \multicolumn{3}{|c|}{$p=0,012$} & \multicolumn{3}{|c|}{$p=0,653$} & \multicolumn{3}{|c|}{$\mathrm{p}=0,400$} \\
\hline
\end{tabular}

Tabla $N^{\circ}$ 4.- Análisis de datos de la encuesta por cargas familiares

En el cansancio emocional se observa que la prueba Chi cuadrado indica dependencia, esto se debe a que en el nivel alto se tiene una mayor proporción que si tienen cargas familiares $(21,9 \%)$.

\begin{tabular}{|c|c|c|c|c|c|c|c|c|c|}
\hline \multirow[b]{3}{*}{ TIENE TRABAJO } & \multicolumn{9}{|c|}{ NIVELES } \\
\hline & \multicolumn{3}{|c|}{ CANSANCIO EMOCIONAL } & \multicolumn{3}{|c|}{ DESPERSONALIZACIÓN } & \multicolumn{3}{|c|}{ REALIZACIÓN } \\
\hline & ВАJO & MEDIO & ALTO & BАJO & MEDIO & ALTO & ВАJO & MEDIO & ALTO \\
\hline SI & $6,0 \%$ & $83,6 \%$ & $10,4 \%$ & $62,7 \%$ & $35,8 \%$ & $1,5 \%$ & - & $58,2 \%$ & $41,8 \%$ \\
\hline NO & $18,8 \%$ & $73,6 \%$ & $7,6 \%$ & $67,4 \%$ & $31,9 \%$ & $0,7 \%$ & - & $69,4 \%$ & $30,6 \%$ \\
\hline TOTAL & $14,7 \%$ & $76,8 \%$ & $8,5 \%$ & $65,9 \%$ & $33,2 \%$ & $0,9 \%$ & - & $65,9 \%$ & $34,1 \%$ \\
\hline PRUEBA CHI & \multicolumn{3}{|c|}{ DEPENDENCIA } & \multicolumn{3}{|c|}{ INDEPENDENCIA } & \multicolumn{3}{|c|}{ INDEPENDENCIA } \\
\hline CUADRADO & \multicolumn{3}{|c|}{$\mathrm{p}=0,047$} & \multicolumn{3}{|c|}{$\mathrm{p}=0,717$} & \multicolumn{3}{|c|}{$\mathrm{p}=0,109$} \\
\hline
\end{tabular}

Tabla $N^{\circ}$ 5.- Análisis de datos de la encuesta por condición laboral 
En el cansancio emocional se observa que la prueba Chi cuadrado indica dependencia, esto se debe a que en el nivel bajo se tiene una mayor proporción que no tienen trabajo $(18,8 \%)$.

\section{Discusión.}

Está claro que el síndrome de Burnout interfiere en gran magnitud en la vida del profesional en todos sus aspectos. Esta investigación servirá de ayuda para los estudiantes y profesionales docentes ya que se pretendió brindar toda la información necesaria para su prevención.

Según, Juárez-García A; et al, 2014, (2) mencionaron que el Burnout, es cada vez más frecuente en nuestra actualidad, pero este tuvo un enfoque principal y es en el personal sanitario donde se da con más frecuencia, debido a que se requiere un contacto directo con las personas.

Castañeda E; García JA, 2012, (3) afirmaron que los odontólogos son los más susceptibles a padecer síndrome Burnout que el resto de personal sanitario, debido al ambiente y forma de trabajo.

Moreno MA; et al, 2005, (4) concordaron que los estudiantes, futuros profesionales estaban sometidos a exigencias académicas rigurosas como su práctica clínica y la preparación de sus clases. Por todo esto, los estudiantes en su proceso de formación están sometidos a una carga de trabajo, donde tendrán que enfrentar a las exigencias para culminar en su carrera.

\section{Conclusiones.}

Los resultados obtenidos mediante esta investigación fueron los siguientes: 
De los datos analizados en esta investigación según el género de los estudiantes no existe ninguna diferencia entre géneros el cansancio emocional se ubica en el nivel medio, despersonalización en nivel bajo y realización profesional en nivel medio.

Según la edad de los estudiantes si existió diferencia, en el grupo de edad de 25 a 30 años existe un nivel alto de cansancio emocional, mientras que en el grupo de edad de 20 a 24 años, el cansancio emocional está en el nivel medio, la despersonalización se coloca para ambos grupos en un nivel bajo y en realización profesional los dos grupos se ubicaron en un nivel medio.

El estado civil marca una diferencia en el cansancio emocional, las personas casadas o en unión libre mostraron niveles altos, mientras que los estudiantes solteros, divorciados, viudos, se establecieron en un nivel medio, la despersonalización para todos los estados civiles se muestra en un nivel bajo, y la realización profesional para todos los estados civiles se manifiesta en un nivel medio.

Las cargas familiares también alteraron los niveles de cansancio emocional, los estudiantes que las tenían presentan un porcentaje alto, los estudiantes que no las poseen están en un nivel medio, en despersonalización no existió diferencia, los dos grupos se presentan en nivel bajo y en realización profesional no existe dependencia los dos grupos presentan porcentajes similares en nivel medio.

Los estudiantes que no poseen un trabajo extra curricular presentan niveles más bajos de cansancio emocional que aquellos que lo poseen, la despersonalización en las dos condiciones no existe diferencia, están en un nivel bajo y en realización profesional los grupos se ubicaron en un nivel medio. 
Dom. Cien., ISSN: 2477-8818

Vol. 3, núm. 1, enero, 2017, pp. 432-442

\section{Bibliografía.}

1. Gil-Monte P. Validez factorial de la adaptación al español del Maslach Burnout Inventory-General Survey. Salud pública Méx. 2002 ene; 44(2): p. 33-40.

2. Juárez-García A, Idrovo A, Camacho-Ávila A, Placencia-Reyes O. Síndrome de burnout en población mexicana: Una revisión sistemática. Salud Ment. 2014 abr; 37(2): p. 159-176.

3. Castañeda E, García J. Prevalencia de Agotamiento Profesional en Odontólogos de una institución educativa y de salud, en la ciudad de Guadalajara, México, en 2012. Guadalajara. Rev Fac Odontol Univ Antioq. 2012 jun; 24(2): p. 267-288.

4. Moreno M, Prado E, García D. Percepción de los estudiantes de enfermería sobre el ambiente De aprendizaje durante sus prácticas clínicas. Revista CUIDARTE. 2013 dic; 4(1): p. 444-449. 